Case Report

\title{
A Fatal Case of Disseminated Intestinal Mucormycosis in a Patient with Vibrio Sepsis
}

\author{
Seungwoo Chung a , Hyun-Jung Sung b, Jong Won Chang c , Ile Hur a,d, Ho Cheol Kim a,d, * \\ ${ }^{a}$ Department of Critical Care Medicine, Gyeongsang University Changwon Hospital, Gyeongsang National University School of Medicine, Changwon, Korea \\ ${ }^{b}$ Department of Pathology, Hanyang University Hanmaeum Changwon Hospital, Changwon, Korea \\ ${ }^{c}$ Department of Surgery, Hanyang University Hanmaeum Changwon Hospital, Changwon, Korea \\ ${ }^{d}$ Department of Internal Medicine, Gyeongsang University Changwon Hospital, Gyeongsang National University School of Medicine, Changwon, Korea
}

\section{Article history:}

Recevied: February 1, 2021

Revised: July 13, 2021

Accepted: July 13, 2021

\section{*Corresponding Author:}

Ho Cheol Kim

Gyeongsang National University Changwon Hospital, 91, Samjeongja-ro, Seongsan-gu, Changwon, Gyeongsangnam 51472, Korea E-mail: hockkim@gnu.ac.kr

\section{ORCID}

Seungwoo Chung

https://orcid.org/0000-0003-3774-6933

Hyun-Jung Sung

https://orcid.org/0000-0002-2533-642X

Ile Hur

https://orcid.org/0000-0002-5832-5233

Ho Cheol Kim

https://orcid.org/0000-0002-3262-0672

\section{Introduction}

Opportunistic infections caused by fungi have increased due to the prevalence of disease such as diabetes mellitus, hematological malignancies, chronic diseases, and a greater number of susceptible patients who have received organ transplants or steroid treatment [1]. The most commonly encountered fungal infections are aspergillosis, mucormycosis, and candida. Mucormycosis is an infection most commonly caused by fungi belonging to the class Zygomycetes, of the order Mucorales, and the genera Rhizopus, Mucor, and Rhizomucor. [2]. Mucorales is found ubiquitously in environments such as soil and rotten trees. It infects the human body through inhalation of fungal sporangiospores or through the mucosa of broken skin [3]. These organisms grow rapidly and produce hyaline septate hyphae in tissue, which can disseminate to other organ systems of the body [4]. Mucormycosis most commonly occurs in the sinus glands (39\%), lungs (24\%), skin (19\%), brain (9\%), gastrointestinal tract (GI, 7\%), and as a disseminated infection (3\%) [5]. The mortality rate is $96 \%$ in disseminated cases, $85 \%$ in GI tract infections, and $76 \%$ in pulmonary infections. Without prompt treatment, mortality rates become very high, with death occurring within $1-2$ weeks $[1,2]$. Since these fungi have a low virulence, mucormycosis mainly occurs in immunocompromised patients, with risk factors such as prolonged and severe neutropenia, malignant hematological diseases with or without stem cell transplantation, poorly controlled diabetes mellitus with or without diabetic ketoacidosis, iron overload, major trauma, prolonged use of corticosteroids, illicit intravenous drug use, neonatal prematurity, use of antifungal agents that have no action against mucorale, such as voriconazole or caspofungin, 
and severe malnourishment [1].

This report discusses a case of mucormycosis infection in the gastrointestinal tract in a patient with nonspecific symptoms and a poor prognosis. We report this case as a concise record of the process of treating such patients.

\section{Case Report}

A 47-year-old woman visited the emergency room with painful blisters around the right ankle and had abdominal discomfort. The patient was diagnosed with Type 2 diabetes mellitus 6 years ago and was taking oral hypoglycemic drugs. In 2017, she was diagnosed, at another hospital, with alcoholic liver cirrhosis which was graded as Child-Pugh B (7-9 points), and she underwent medical treatment. On arrival hospital, the patient's vital signs were blood pressure 80/50 $\mathrm{mmHg}$, heart rate 92 beats per minute, respiratory rate 20 breaths per minute, body temperature $36.2^{\circ} \mathrm{C}$, and the initial blood test findings were as follows: white blood cell count

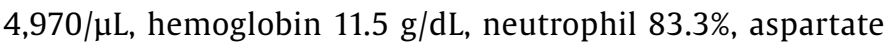
transaminase/alanine transaminase 164/26 IU/L, albumin $2.5 \mathrm{~g} /$ $\mathrm{dL}$, total bilirubin $5.87 \mathrm{mg} / \mathrm{dL}$, blood urea nitrogen $30.4 \mathrm{mg} / \mathrm{dL}$, creatinine $2.80 \mathrm{mg} / \mathrm{dL}, \mathrm{pH} 7.375, \mathrm{pCO} 226.1 \mathrm{mmHg}, \mathrm{pO} 275.2$ $\mathrm{mmHg}$, bicarbonate $15.3 \mathrm{mmol} / \mathrm{L}$, base excess -9.9 , lactate 78.9 $\mu \mathrm{mol} / \mathrm{L}, \mathrm{C}$-reactive protein $3.12 \mathrm{mg} / \mathrm{dL}$. The patient complained of abdominal discomfort, however, the abdominal computed tomography (CT) revealed no specific findings other than gall bladder stones. Prior to the patient losing consciousness and showing signs of delirium, the patient had reported eating raw fish 3 days before the visit to the hospital. She was started on a continuous infusion of norepinephrine and she was administered $2 \mathrm{~g}$ of ceftriaxone, and $300 \mathrm{mg}$ of clindamycin. She was diagnosed with septic shock caused by vibrio infection. Later, the patient was admitted to the intensive care unit and was managed by the Gastroenterology Department.

The patient was not resuscitated despite continued administration of norepinephrine, so a dopamine infusion was added. Since there was oliguria after admission, continuous renal replacement therapy was started on the 2nd day of hospitalization. Vibrio vulnificus was identified in the blood culture that was performed during the emergency room visit. Urine flow resumed and continuous renal replacement therapy was stopped on the 4th day of hospitalization. However, she remained in shock; the administration of vasopressors, total parenteral nutrition, and fasting were maintained.

Follow-up abdominal CT scans were performed 8 days after hospitalization and showed bowel ileus due to colitis, and conservative treatment was continued. The patient still showed symptoms of delirium and complained of sudden, severe abdominal tenderness on the 13th day of hospitalization. An abdominal CT scan revealed an ascending colon perforation, and emergency surgery was performed on the same day. Ascending colon perforation and panperitonitis were noted during the surgery, a right hemicolectomy and an ileocolic anastomosis were performed (Figure 1).

The patient was taken off the mechanical ventilator on the day after surgery, but she remained in shock. No bacteria were identified in an additional blood culture, but she developed a fever on the 13th day of hospitalization. Catheter tip culture was performed after central venous catheter removal; teicoplanin administration was started due to the suspicion of catheter related bloodstream infections. Pathological examination of the surgical specimen confirmed mucormycosis. It was Day 16 of hospitalization. Liposomal amphotericin (5 mg/kg/day) administration was started and teicoplanin administration was discontinued because there was no bacterial infection detected.

Despite the administration of liposomal amphotericin, the patient's condition did not improve; dyspnea and acidosis worsened. Intubation and continuous renal replacement therapy were resumed on the 23rd day of hospitalization. However, the patient died on the 25th day of hospitalization.

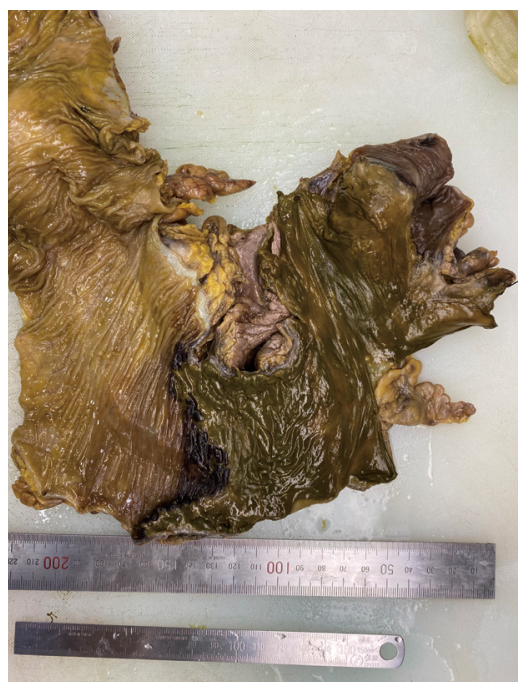

Figure 1. Grossly, the purulent mucosa necrosis is located in distal ascending colon segmentally, resembling transmural infarct necrosis.

\section{Discussion}

GI mucormycosis is primarily caused by the ingestion of fungi for example through food or contaminated sputum. The prevalence of mucormycosis infections in the GI tract is in the following order: stomach (57.7\%), colon (23.3\%), ileum (7\%), esophagus (7\%), duodenum (2.3\%), and jejunum (1.1\%) [2]. Clinical symptoms vary depending on the site of infection. The first manifestations are mainly non-specific abdominal pain, abdominal distension, fever, and diarrhea. Tissue infarction 
(A)

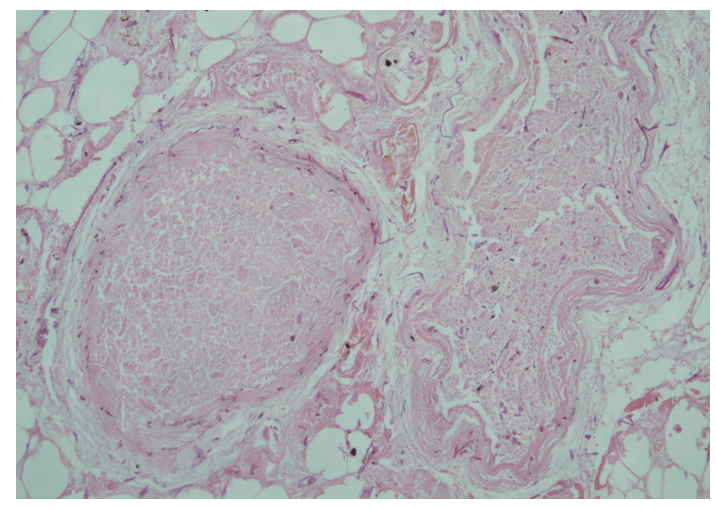

(B)

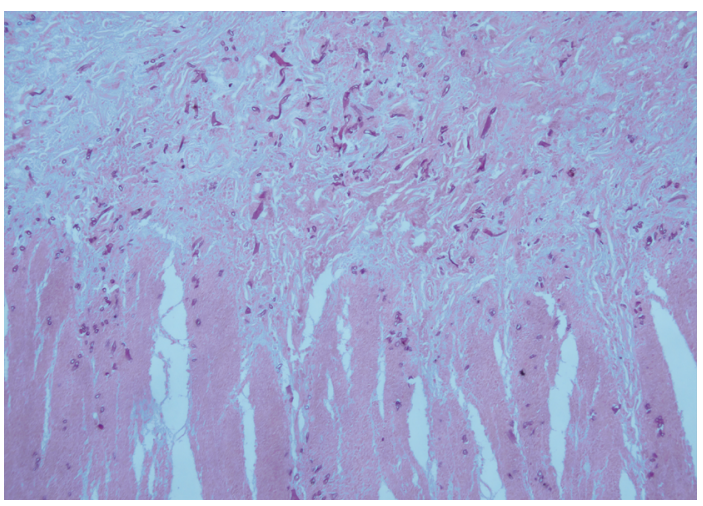

Figure 2. (A) Microscopically, the lesion shows transmural coagulative necrosis due to acute vascular ischemia with disseminated fungal infection and frequent angioinvasion. $x$ 200. (B) The fungi show broad, non-septate hyphae with wide angle branching. $x 100$.

and necrosis due to vascular invasion and blood clots strongly suggest the possibility of hematogenous dissemination. Death maybe caused by intestinal bleeding, perforation, peritonitis, and sepsis [6].

GI mucormycosis is a rare infection, but its incidence has been increasing in Korea in recent years as the number of immunocompromised patients with chronic renal failure, kidney transplantation, hematological malignancy, or alcoholic liver cirrhosis has increased [7]. There are only a few reports of successful treatment for invasive GI mucormycosis due to the high mortality rate. Ryu et al [7] reported a case of gastric ulcer perforation in an alcoholic patient with liver cirrhosis. The patient underwent a subtotal gastrectomy and gastrojejunostomy. Pathological examination determined the diagnosis of gastric mucormycosis and was administered liposomal amphotericin B ( $5 \mathrm{mg} / \mathrm{kg} /$ day); he survived and was discharged from hospital. Ha et al [8] reported a case of GI mucormycosis in a patient with idiopathic cyclic neutropenia. The 31-year-old male underwent surgery due to repeated bowel perforations; he was diagnosed with mucormycosis following a pathological examination, and was administered liposomal amphotericin B ( $5 \mathrm{mg} / \mathrm{kg} /$ day), but he did not survive. Compared with other reports, the patient in the Case Report is a rare case of mucormycosis with vibrio infection.

Therefore, among immunocompromised patients in whom vital signs deteriorate without response to the appropriate antibiotics, opportunistic fungal infections and especially mucormycosis should be included in the differential diagnosis. Unlike other bacterial infections, there is no reliable serological test or skin test for the diagnosis of mucormycosis. If in case of decreased attenuation of the intestinal wall in CT or magnetic resonance imaging tests, due to bowel edema, infarction, or necrosis, or if there is pneumoperitoneum and pneumatosis intestinalis due to ischemia or perforation, they should be considered as helpful pointers for diagnosis. After tissue biopsy

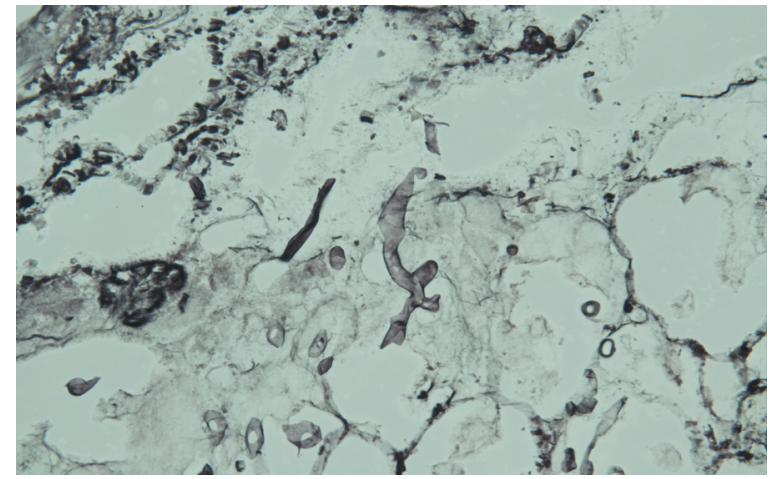

Figure 3. Gomori's methenamine-silver stain demonstrating branched wide angled fungal hyphae. $x 400$.

is performed on the suspected area by surgery or endoscopy, it can be confirmed under microscope by wide angled large, nonsepta hyphae- a characteristic histopathological examination finding (Figures 2 and 3) [9].

The patient in this case, who had diabetes mellitus and liver cirrhosis as underlying diseases, received critical care in the intensive care unit for shock from vibrio sepsis, but did not recover. The abdominal CT taken on the 8th day of admission showed only nonspecific ascending colitis, and the followup abdominal CT taken on the 13th day of hospitalization showed pneumoperitoneum. The surgical findings revealed an ascending colon perforation with necrotic changes observed in the mesentery, terminal ileum, and ascending colon. The small intestine and other mesentery did not show any abnormalities other than inflammation due to panperitonitis. The pathological examination revealed transmural infarction with mucormycosis and multiple vascular invasions. Thrombus was observed in the ileocolic artery, which led to ischemic changes at the proximal end of the ascending colon. The location of the bowel perforation was 1 meter away from the vascular infarction lesion that was due to mucomycosis. Therefore, it is presumed that mesenteric ischemia occurred due to vascular invasion by mucorale; this 
could not be ruled out as a result of the use of high doses of vasopressor.

GI mucormycosis progresses quickly and the angioinvasion frequency is high, which leads to blood clots and tissue necrosis. This is the main reason why the antifungal agent does not effectively penetrate the infected sources. Rapid surgical debridement of infected lesions can increase survival rates. Mucormycosis requires both surgical treatment and antifungal administration rather than antifungal therapy alone [9]. Currently, the recommended antifungal treatments for mucormycosis are amphotericin B (1-1.5 mg/kg/day) and liposomal amphotericin B (5 mg/kg/day) $[9,10]$. In refractory mucormycosis which does not improve even after the above treatments, posaconazole can be administered as salvage therapy [10]. Posaconazole has high efficiency when taken with food, but its bioavailability is known to vary greatly depending on gastrointestinal disorders and food intake. In addition to this, it is necessary to treat the underlying condition of the patient and correct the factors that lower immunity.

The patient in this case was diagnosed with disseminated mucormycosis with angioinvasion and was started on liposomal amphotericin B ( $5 \mathrm{mg} / \mathrm{kg} /$ day). Following treatment, she showed short-term improvement, but did not recover from fungal sepsis. Posaconazole was considered for refractory mucormycosis, but oral posaconazole could not be administered as high dose vasopressors were already being administered. In addition, prolonged periods of nil per os such as in this patient, increase the prevalence of opportunistic infections and decrease the patient's immunity through mechanisms such as reduced normal flora. Therefore, active enteral nutritional support in patients with stable vital signs will have favorable effects on their prognosis.

As reports of mucormycosis have continued to increase in recent years, we should be able to start rapid diagnosis and treatment by including mucromycosis in the differential diagnosis when immunocompromised patients, with various chronic underlying diseases, do not respond well to existing treatments such as antibiotics, and surgery. Moreover, when the results of such case reports are gathered, a guideline for appropriate treatment can be prepared.

\section{Conflicts of Interest}

The authors have no conflicts of interest to declare.

\section{Ethical Statement}

This research did not involve any human or animal experiment.

\section{References}

[1] Petrikkos G, Skiada A, Lortholary O, Roilides E, Walsh TJ, Kontoyiannis DP. Epidemiology and clinical manifestations of mucormycosis. Clin Infect Dis 2012;54 Suppl 1:S23-34.

[2] Roden MM, Zaoutis TE, Buchanan WL, Knudsen TA, Sarkisova TA, Schaufele RL, et al. Epidemiology and outcome of zygomycosis: A review of 929 reported cases. Clin Infect Dis 2005;41(5):634-53.

[3] Petrikkos G, Drogari-Apiranthitou M. Zygomycosis in immunocompromised non-haematological patients. Mediterr J Hematol Infect Dis 2011;3(1):e2011012.

[4] Prabhu RM, Patel R. Mucormycosis and entomophthoramycosis: A review of the clinical manifestations, diagnosis and treatment. Clin Microbiol Infect 2004;10 Suppl 1:31-47.

[5] Agha FP, Lee HH, Boland CR, Bradley SF. Mucormycoma of the colon: Early diagnosis and successful management. Am J Roentgenol 1985;145(4):73941.

[6] Lo OSH, Law WL. Ileocolonic mucormycosis in adult immunocompromised patients: A surgeon's perspective. World J Gastroenterol 2010;16(9):116570.

[7] Lee SH, Son YG, Sohn, SS, Ryu SW. Successful treatment of invasive gastric mucormycosis in a patient with alcoholic liver cirrhosis: A case report. Exp Ther Med 2014;8(2):401-4.

[8] Ha TS, Park CM, Yang JH, Cho YH, Chung CR, Jeon K, et al. Disseminated Gastrointestinal Mucormycosis in Immunocompromised Disease. Korean J Crit Care Med 2015;30(4):323-8.

[9] Cornely OA, Arikan-Akdagli S, Dannaoui E, Groll AH, Lagrou K, Chakrabarti A, et al. ESCMID and ECMM joint clinical guidelines for the diagnosis and management of mucormycosis 2013. Clin Microbiol Infect 2014;20 Suppl 3:5-26.

[10] Tacke D, Koehler P, Markiefka B, Cornely OA. Our 2014 approach to mucormycosis. Mycoses 2014;57(9):519-24. 